\title{
Stress and psychiatric disorder in urban Rawalpindi
}

\author{
Community survey
}

DAVID B. MUMFORD, FAREED A. MINHAS, IMTIAZ AKHTAR, SAEED AKHTER and MALIK H. MUBBASHAR

\begin{abstract}
Background Recent studies in rural areas of Pakistan have yielded high prevalence rates of common mental disorders, especially among women.
\end{abstract}

Aims To investigate emotional distress and common mental disorders in a poor urban district using the same survey method.

Method First-stage screening of a slum district of Rawalpindi used the Bradford Somatic Inventory. Psychiatric interviews were conducted with stratified samples using the ICD-10 research diagnostic criteria.

Results On a conservative estimate, $25 \%$ of women and $10 \%$ of men suffered from anxiety and depressive disorders.

Levels of emotional distress increased with age in both men and women. Women living in joint households reported more distress than those living in unitary families. Higher levels of education were associated with lower risk of common mental disorders, especially in younger women. Emotional distress was negatively correlated with socio-economic variables among women.

Conclusions This study found levels of emotional distress and psychiatric morbidity in a poor district of Rawalpindi to be less than half those in a nearby rural village in the Punjab, although rates in women were still double those in men. Possible explanations are that more healthy people migrate to the cities or that urban living is more conducive to good mental health in Pakistan.

Declaration of interest None.
Two recent epidemiological surveys of rural populations in Pakistan - in the mountains of Chitral and on the plains of the Punjab both found very high rates of common mental disorders, especially among women (Mumford et al, 1996, 1997). There have been no previous community-based studies of urban populations in Pakistan. Elsewhere on the Indian subcontinent, Sethi et al (1974) reported rates of non-psychotic mental disorders of 27 per 1000 adults in the city of Lucknow, compared with 20 per 1000 in the surrounding rural area. Dube (1970) also reported rates of neurosis about one-third higher in the city of Agra than in nearby rural areas. The aims of the present study were to estimate the prevalence of common mental disorders in an urban slum area of Pakistan, to identify social factors and stressors that correlate with emotional distress and to compare these results with those of the Chitral and rural Punjab studies.

\section{METHOD}

\section{Population studied}

Like other cities of Pakistan, Rawalpindi has grown enormously in the past 50 years, mainly by migration from the surrounding villages, and is now a sprawling city of 900000 people. There is a wide variation in socio-economic status and some areas of the city lack even the basic amenities of piped water, sewerage and electricity. Sultanpura, the district selected for this study, is a typical heavily populated slum area. By origin it was not a planned residential area, but was incorporated into Rawalpindi city by the municipal authorities only about 10 years ago, when the construction of roads, electricity, water and gas supply and a drainage system were begun.

Before starting the study, the local councillor and other notables were approached to secure their cooperation and support.
The study population was defined as all persons aged 18 years and over living in Mohalla Sultanpura: 1210 persons, according to Municipal Corporation statistics. A sketch map of the area was drawn, streets and plots were identified and each house was assigned a unique study number.

\section{The Bradford Somatic Inventory}

In a south Asian context, if a screening instrument is based primarily on psychological symptoms, many cases of neurosis are likely to be missed. Most people with common mental disorders spontaneously express their experience not in psychological symptoms but in somatic complaints. The Bradford Somatic Inventory (BSI; Mumford et al, 1991) was developed from symptom reports by psychiatric patients in Pakistan and Britain with clinical diagnoses of anxiety or depression. The BSI enquires about a wide range of somatic symptoms during the previous month and, if the subject has experienced a particular symptom, whether the symptom has occurred on more or less than 15 days during the month (scoring 2 or 1 , respectively). In two recent epidemiological surveys in Pakistan (Mumford et al, 1996, 1997) the BSI proved to be an effective first-stage screening instrument, as judged by subsequent selective psychiatric interviews using ICD-10 research diagnostic criteria (World Health Organization, 1993). Test-retest reliability of the BSI administered after an interval of a week was good, with an overall reliability coefficient of 0.86 and a median $\kappa$ value of 0.63 in a British primary care population (Mumford, 1992).

\section{First stage}

The epidemiological survey was conducted in two stages: screening questionnaire and psychiatric interviews. Four survey workers for the first stage were recruited from among the paramedical staff at the Department of Psychiatry, Rawalpindi General Hospital. They were trained in the administration of the BSI and a household pro forma in a 3-day training programme. Each worker achieved satisfactory interrater reliability standards: the overall BSI item agreement was $95 \%$.

At each house, a list was compiled of all the adults and children, recording their name, gender, age, marital status, relationship to the head of the household and years of education. After obtaining consent, the 
BSI-44 was administered orally in Urdu to each person aged 18 years and over. Male subjects were interviewed by male raters and female subjects by female raters; some degree of privacy was attempted.

Raters revisited many houses to complete the administration of questionnaires for all the adult members of the household, in order to minimise bias in recruitment. Subjects suffering from acute or chronic psychotic disorders or showing evidence of cognitive impairment were identified and excluded.

\section{Second stage}

Psychiatric assessments were undertaken on subjects scoring above the cut-off point on the BSI-44 and on a random sample of subjects scoring below this threshold. The choice of cut-off point was determined in a pilot study of psychiatric out-patients at Rawalpindi General Hospital with diagnoses of anxiety or depressive disorders. The psychiatric interviews were conducted without knowledge of the BSI scores obtained in the first stage.

The interview consisted of two elements: the identification and diagnosis of any psychiatric disorder according to ICD-10 research diagnostic criteria, using a psychiatric assessment schedule administered in Urdu/Punjabi, and the identification of any concurrent physical illness (whenever this seemed a possibility, a physical examination was carried out).

The psychiatric assessment schedule utilised selected ('double asterisked') questions from the Present State Examination (PSE; Wing et al, 1974) as screening items, followed by the appropriate criteria from the ICD-10 research diagnostic criteria. The psychiatric assessment systematically enquired about the following disorders (in hierarchical order): depressive episode, dysthymia, obsessive-compulsive disorder, panic disorder, agoraphobia, social phobia, specific phobias, generalised anxiety disorder and somatisation disorder. This diagnostic hierarchy reflects the ICD-10 research diagnostic criteria and thereby avoids dual diagnoses.

The interviews were conducted by I.A. and S.A., both trainee psychiatrists, who received a 4-day training programme in the use of the psychiatric assessment schedule, achieving an item concordance rate of $90 \%$. During the study, psychiatric diagnoses were made after discussion between the interviewer and F.A.M.
After administering the psychiatric assessment schedule, the interviewer used the Socio-Economic Scales for Pakistan (SESP; Mumford \& Mirza, details available from D.B.M. upon request) to code household income, sanitation and plumbing, electrical appliances and family wealth in land and livestock. Other households were visited separately to complete the SESP. The interval between the first and second stages of the survey was kept to less than 7 days, particularly to avoid losing subjects who might leave the area.

\section{RESULTS}

\section{Demography}

Between April and August 1998, 240 households were surveyed: $98 \%$ of the total number in Sultanpura. Five houses were excluded because the residents were away for the whole period of the study. There were 774 adults ( 408 men and 366 women) and 612 children aged under 18 years: current residence in the household was defined as sleeping in the house most nights each week. Family members working or studying elsewhere in Pakistan were not included. The excess of men (mainly in the 30-60year age band) represents men lodging in the city for work, having left their families behind in their home villages. Overall, $67 \%$ of the adults were married, $29 \%$ were single and $4 \%$ were widowed. Adult literacy rates were $69 \%$ for males and $52 \%$ for females.

There was a great diversity of family structure in Sultanpura, which was difficult to categorise simply. The 240 households were grouped broadly into unitary (parents and children only, or a married couple without children), extended (three generations), joint (with married sons or daughters, their spouses and children), polygamous and male-only households: $78 \%$ of the households were unitary, $4 \%$ were extended, $13 \%$ were joint households, $2 \%$ were polygamous and $3 \%$ were maleonly shared apartments. The mean household size was 3.2 adults and 2.6 children under age 18 years. The socio-economic profile of households is given in Table 1 .

\section{First stage}

The BSI-44 was administered in Urdu to 760 adults, representing $98 \%$ of the total adult population in the study frame. Of the fourteen subjects not included, five were excluded on medical grounds (dementia,
Table I Socio-economic profile of households

\begin{tabular}{|c|c|}
\hline \multicolumn{2}{|l|}{ Occupation of head of household } \\
\hline Skilled clerical workers or in trade & $35 \%$ \\
\hline Craftsmen or skilled industrial workers & $7 \%$ \\
\hline Unskilled workers & $25 \%$ \\
\hline Professional/business & $7 \%$ \\
\hline Retired or pensioners & $3 \%$ \\
\hline Unemployed & $1 \%$ \\
\hline \multicolumn{2}{|l|}{ Education of head of household } \\
\hline No formal education & $36 \%$ \\
\hline $\mathrm{I}-5$ years & $15 \%$ \\
\hline $6-9$ years & $12 \%$ \\
\hline$\geqslant 10$ years & $35 \%$ \\
\hline \multicolumn{2}{|l|}{ Education of children } \\
\hline Government Urdu-medium school & $40 \%$ \\
\hline Private Urdu-medium school & $23 \%$ \\
\hline Private English-medium school & $7 \%$ \\
\hline \multicolumn{2}{|l|}{ Household income } \\
\hline$<1000$ rupees $(£ 20)$ per month & $5 \%$ \\
\hline $1000-5000$ rupees $(£ 20-100)$ per month 5 & $55 \%$ \\
\hline $\begin{array}{l}5000-10000 \text { rupees }(£ 100-200) \text { per } \\
\text { month }\end{array}$ & $30 \%$ \\
\hline$>10000$ rupees $(£ 200)$ per month & $11 \%$ \\
\hline \multicolumn{2}{|l|}{ House ownership } \\
\hline Owned & $65 \%$ \\
\hline Rented & $35 \%$ \\
\hline \multicolumn{2}{|l|}{ Plumbing } \\
\hline Inside toilet & $99 \%$ \\
\hline Closed drains & $83 \%$ \\
\hline Hot water system & $35 \%$ \\
\hline \multicolumn{2}{|l|}{ Ownership of domestic electrical appliances } \\
\hline Electric fan & $98 \%$ \\
\hline Electric iron & $82 \%$ \\
\hline Radio & $61 \%$ \\
\hline Cassette recorder & $50 \%$ \\
\hline Black-and-white television & $30 \%$ \\
\hline Colour television & $39 \%$ \\
\hline Refrigerator & $43 \%$ \\
\hline \multicolumn{2}{|l|}{ Ownership of land } \\
\hline None & $71 \%$ \\
\hline$<$ I acre & $18 \%$ \\
\hline $1-10$ acres & $7 \%$ \\
\hline$>10$ acres & $4 \%$ \\
\hline \multicolumn{2}{|l|}{ Ownership of vehicles } \\
\hline Pedal bicycle & $36 \%$ \\
\hline Motor bicycle & $31 \%$ \\
\hline Car & $7 \%$ \\
\hline
\end{tabular}

deafness, serious physical illness), four refused to participate and five subjects were unavailable every time the raters called at their houses. Figure 1 shows the mean scores on the BSI by gender and age band: women had significantly higher BSI scores 


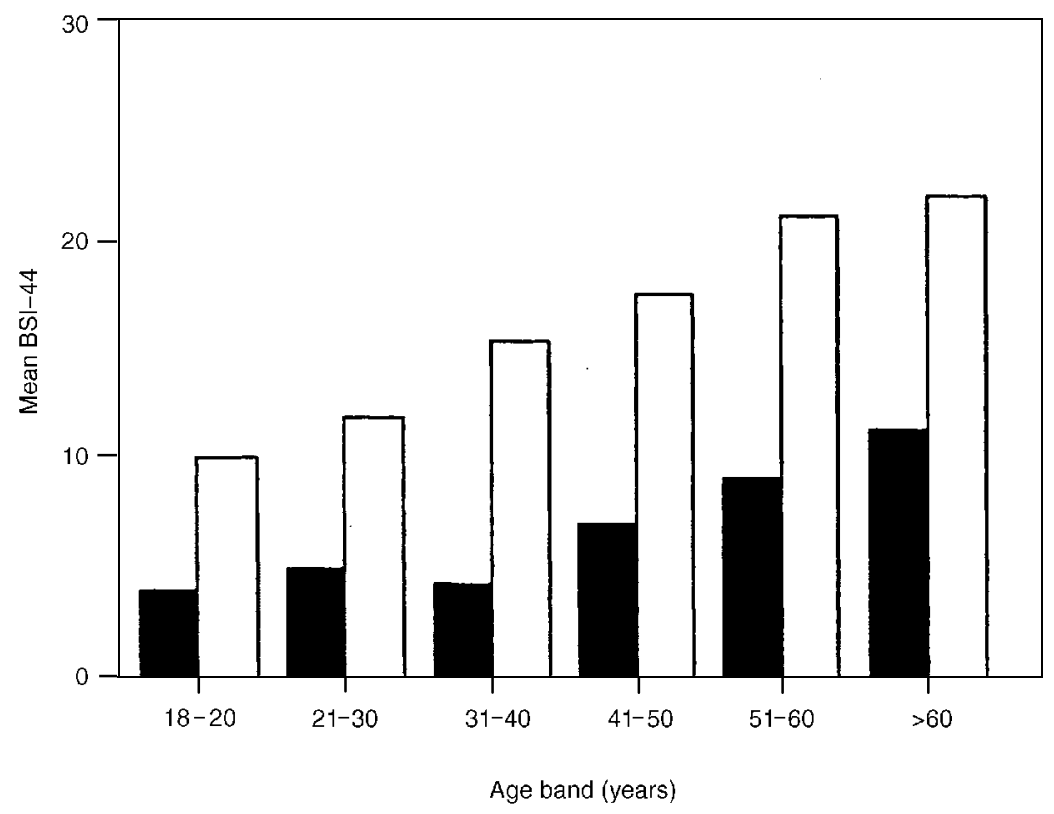

Fig. I Mean Bradford Somatic Inventory scores by age band and gender: $\square$ male; $\square$ female.

than men in every age band; $28 \%$ of women and $11 \%$ of men had BSI scores above the 20/21 threshold.

\section{Second stage}

It was decided to interview subjects scoring 21 or above on the BSI and also a 1 in 10 sample of subjects scoring 20 or below, chosen using random number tables. On this basis, 220 subjects (138 women and 82 men) were selected: interviews were conducted with 215 (98\%). Five subjects could not be traced at the time of the interview; no one declined to be interviewed. The numbers of men and women receiving psychiatric diagnoses at interview are given in Table 2.

\section{Estimated prevalence of psychiatric disorders}

The point prevalence of non-psychotic psychiatric disorders, based only on subjects with BSI scores of 21 and above, was $25 \%$ for women and $10 \%$ for men. The estimate of prevalence, including subjects scoring in the sub-threshold range of BSI scores (weighted to allow for the 1:10 sampling ratio), rises to $36 \%$ for the women and $16 \%$ for men. In addition, $1 \%$ of subjects received other psychiatric diagnoses (three cases of dementia, one of learning disability and one of acute psychotic illness, cannabis-induced).
Using the 20/21 threshold, the sensitivity of the BSI-44 in this study was $65.5 \%$, specificity was $98.6 \%$, the positive predictive value (PPV) was $94.2 \%$ and the negative predictive value (NPV) was $88.8 \%$, after weighting for the actual sampling ratio of sub-threshold subjects. The sensitivity, specificity, PPV and NPV of the BSI were similar between men and women.

\section{Bradford Somatic Inventory scores and socio-demographic factors}

Using the BSI as a general measure of emotional distress, the relationships between BSI scores and demographic and socio-economic variables were explored, including age, gender, marital status, educational status, household income and family wealth.

\section{Demographic variables}

Age. Figure 1 shows that mean BSI scores increase with age in both men and women, although the pattern is different. In women, there is a steady rise from age 18-20 years onwards, reaching a plateau from the age of 50 years. With men, there is a plateau from age 18 to 40 years and a steady rise thereafter. Analysis of variance of BSI scores confirmed the effects for both gender $(F=83.2, P<0.001)$ and age band $(F=8.3$, $P<0.001$ ), but the two-way interaction
Table 2 Psychiatric ICD-10 diagnoses at interview

\begin{tabular}{lrr}
\hline ICD-10 diagnosis & Male & Female \\
\hline Depressive episode, severe & 7 & 23 \\
Depressive episode, moderate & 16 & 27 \\
Depressive episode, mild & 8 & 18 \\
Dysthymia & 3 & 1 \\
Panic disorder & 1 & 4 \\
Agoraphobia & 1 & 3 \\
Social phobia & 1 & 2 \\
Generalised anxiety disorder & 6 & 15 \\
Somatisation disorder & 0 & 0 \\
Obsessive-compulsive disorder & 0 & 4 \\
No psychiatric disorder & 37 & 38 \\
& & \\
Total interviews & 80 & 135 \\
\hline
\end{tabular}

was not significant. Because of this strong effect of gender and age on BSI scores, all subsequent analyses using BSI scores are controlled for gender and age.

Marital status. Mean BSI scores were almost identical between single and married women aged 20-30 years, and the same applied in men of this age. Widows aged 30-50 years had higher mean BSI scores than married women of the same age (23.6 v. 15.2), but this difference did not reach statistical significance because there were only eight widows.

Household type. For men, an analysis of variance of BSI scores on household type showed no significant difference overall, nor when heads of household, sons or brothers were analysed separately. However, for women there was a significant overall variance in BSI scores by household type $(n=363, F=3.437, P<0.001)$, also found separately with wives of the heads of household $(n=216, F=4.478$, $P<0.0001)$, their daughters $(n=77$, $F=3.406, P=0.008)$ and mothers $(n=19$, $F=7.082, \quad P=0.002$ ), although not with daughters-in-law. These effects remained significant after age had been included as a covariate.

Among wives of the heads of household, the highest mean BSI scores were found in joint households $(20.9, n=28)$, polygamous households $(19.1, n=8)$ and 'mature' unitary families where the majority of offspring were adults, all 
unmarried (18.3, $n=28)$; the lowest mean BSI scores were found with married couples without children (4.2, $n=11)$. Among their daughters, much higher mean BSI scores were found in joint households (23.4, $n=16)$ than in unitary families $(8.6$, $n=60$ ). Among mothers of the heads of household, mean BSI scores were much higher in joint households than in unitary or extended households. Women living in standard nuclear households generally had lower than average BSI scores.

Years ofeducation. Subjects were categorised according to the number of years of formal education received (nil, 1-5 years, 6-9 years and 10+ years). Lower levels of education were associated with higher BSI scores. In an analysis of variance of BSI scores, educational category was a highly significant factor $(F=4.35, P=0.005)$ alongside age $(F=4.03, P=0.001)$ and gender $(F=78.7, P<0.001)$. The beneficial effect of education was most marked among younger women aged 18-40 years $(F=2.75, P<0.05)$. The same trend was found in both younger and older men. Among older women (aged 41 years and over) there was no relationship between BSI scores and educational category, but $83 \%$ of women in this age group had had no formal education.

\section{Socio-economic factors}

There were significant negative correlations between individual BSI scores and several socio-economic variables (total household income, education of the head of the household, ownership of a motor vehicle). The magnitude of the correlation coefficients differed between men and women. Among men, BSI scores correlated most strongly with household income $(-0.11)$; among women, the strongest correlations were with household income $(-0.20)$, number of electrical appliances $(-0.14)$ and education of the head of the household $(-0.12)$ (in every case, $P<0.05$ ).

An exploratory factor analysis was used to condense these socio-economic variables into one or more simpler summary factors. Cattell's screen test (Cattell, 1966) suggested the extraction of only one factor, which was obtained using a principal components analysis. This 'general wealth' factor had high loadings for total household income (0.81), number of electrical appliances (0.75), plumbing facilities (0.68), ownership of motor vehicles $(0.60)$, number of rooms in the house (0.51) and type of education given to the children $(0.46)$. The total variance explained was $38 \%$. The general wealth factor correlated -0.08 with BSI scores overall $(P=0.02)$ : correlations were much higher in women $(-0.12)$ than in men $(-0.03, \mathrm{NS})$.

\section{Socio-demographic factors in combination}

A stepwise multiple linear regression analysis was conducted with BSI scores as the dependent variable and age, years of education and the general wealth factor as predictor variables. With both men and women, age and years of education (but not general wealth) independently contributed to the variance in BSI scores: the total variance explained was $6-7 \%$.

\section{DISCUSSION}

This study is the third in a series of community-based epidemiological surveys of common psychiatric disorders in Pakistan using the same methodology. The previous studies, in the mountain villages of Chitral and in the Punjab, were of rural populations (Mumford et al, 1996, 1997). The Punjab study in particular, conducted in a village less than $50 \mathrm{~km}$ from Rawalpindi, allows direct comparison between urban and rural populations in the same region.

The most striking finding from the present study is the much lower rate of psychiatric morbidity in this urban slum area than in the rural areas surveyed previously (Table 3). This was unexpected. This finding applies to both men and women, although women have much higher rates of morbidity than men in all three studies.

Table 3 Estimated prevalence of common mental disorders

\begin{tabular}{lccc}
\hline & $\begin{array}{c}\text { Chitral, } \\
\text { Northern } \\
\text { Pakistan }\end{array}$ & $\begin{array}{c}\text { Susral village, } \\
\text { Gujar Khan, } \\
\text { Punjab }\end{array}$ & $\begin{array}{c}\text { Sultanpura, } \\
\text { Rawalpindi }\end{array}$ \\
\hline Men & $15-33 \%$ & $25-44 \%$ & $10-16 \%$ \\
Women & $46-60 \%$ & $66-72 \%$ & $25-36 \%$ \\
\hline
\end{tabular}

An estimated prevalence range is given: the lower figure has been calculated conservatively based only on subjects in the high Bradford Somatic Inventory (BSI) scoring categories who met ICD-10 research diagnostic criteria for a psychiatric disorder; the higher figure also includes an estimate of rates of ICD-10 psychiatric disorders in subjects with BSI scores in the subthreshold range.

\section{Urban versus rural populations}

In Western societies, poor urban populations have generally been found to exhibit higher rates of morbidity than rural communities (Harpham, 1992). Brown \& Harris (1978) reported the 1-month prevalence of all psychiatric illness as $17 \%$ among women in Camberwell, London, compared with a 1-year prevalence of $12 \%$ among women in North Uist, Outer Hebrides (Brown \& Prudo, 1981). The Epidemiologic Catchment Area (ECA) study (Regier \& Kaelber, 1995) found the 1-year prevalence of major depression in an urban area in North Carolina to be more than twice that of a remote and isolated rural area, although in St Louis the prevalence of depression was lower in the urban centre than in an adjacent rural area connected to it by suburban sprawl.

In urban areas in the developing world, poor social conditions in the rapidly growing cities may be responsible for high rates of stress and psychiatric morbidity (Mari, 1987; Desjarlais et al, 1995). Almeida-Filho (1987) reviewed many epidemiological studies conducted in Latin America that have sought to assess the impact of 'urban stress' and the effects of economic and social marginalisation on the mental health of rural migrants to the cities.

However, there have been few paired studies of rural and urban populations in the same geographical region to allow direct comparisons. An epidemiological study in Puerto Rico found higher prevalence rates of most DSM-III-defined (American Psychiatric Association, 1980) psychiatric disorders among urban than rural residents (Canino et al, 1987). In South Korea, Lee et al (1990) found no difference in rates of major psychiatric disorders between urban and rural Seoul. In Taiwan, 'small town' samples showed significantly higher rates for most nonpsychotic disorders than 'rural villages', but both were higher than in modern metropolitan Taipei (Hwu et al, 1989). However, in another study of urbanisation in Taiwan, Cheng (1989) found higher rates of depression among young women in rural villages than in urban or suburban areas, associated with chronic stressors, mostly around difficulties in family relationships.

Other explanations for observed differences between urban and rural populations must be considered. Guarnaccia et al (1990) have warned of the effects of an 
acquiescent response style on self-report symptom measures, resulting in inflated prevalence estimates. Urban populations may be more sophisticated in their response style than rural people. However, in all three studies in Pakistan, the BSI questionnaires were followed by selective psychiatric interviews using ICD-10 research diagnostic criteria (on which the prevalence estimates were based), which seem to be less susceptible to this kind of bias. The sensitivity and specificity of the BSI with respect to ICD-10 diagnoses were similar in all three studies.

\section{Socio-demographic factors and stress}

The pattern of BSI scores with respect to age and gender (Fig. 1) was identical to that in the rural Punjab and very similar to that in Chitral. Women have much higher BSI scores than men in every age group. In all countries of the world, women experience higher levels of psychiatric morbidity than men, but the gap appears greatest in poorer countries (Lee et al, 1990; Desjarlais et al, 1995). Life may be particularly stressful for most women in Pakistan because of their lack of control over their lives.

One notable finding in Sultanpura was the great variety of domestic family patterns, making the classification of household types not at all straightforward. Whereas in the rural studies households fell quite neatly into three categories (joint, extended and unitary), in the urban setting almost any permutation and variation was found. This reflects the splitting up of families into different living groups, often with part of the joint/extended family still living back in the ancestral village. These fragmented and shifting family living arrangements seem to be the direct social consequence of ruralurban migration.

This study found that women are much more affected than men by the type of household and their place within the family. Women from joint households and 'mature unitary' families showed especially high mean BSI scores. Polygamy seems bad for women's mental health but good for men's - like marriage in general, only more so!

All three of these studies used the same set of socio-economic scales for Pakistan, which included a wide range of potential indicators of socio-economic status relevant to both rural and urban communities.
Higher levels of emotional distress (as measured by BSI scores) were significantly associated with poorer socio-economic status in Chitral, in rural Punjab and in urban Rawalpindi, although the most significant indicators of socio-economic status were different. In rural societies, wealth is measured by ownership of land and livestock, whereas in the cities household income is the most important factor. The educational level achieved and the number of electrical appliances were useful indicators of socio-economic status in both urban and rural settings. In Sultanpura, as in rural Punjab and in Chitral, socioeconomic status has more impact on the mental health of women than of men.

\section{Migration and mental health}

The present study has confounded our expectations and found much lower rates of common psychiatric disorders in the urban slum area of Sultanpura than in the rural populations of Punjab and Chitral. What light might this shed on the social processes and psychiatric consequences of the worldwide phenomenon of migration from rural areas of developing countries into the cities?

Sultanpura is a comparatively recent urban settlement and is typical of a certain kind of peripheral urban development, but it is not representative of the whole city. Most existing householders were the primary migrants and had built their own houses there. Most had migrated from the surrounding rural areas of the Punjab. We enquired of a sample of these householders why they had chosen to move to the city. Most gave one of three reasons: poverty and lack of work in the village; to obtain better education for their children; or because of family quarrels, particularly in a joint family system.

Since Odegaard's pioneering study of Norwegians migrating to Minnesota (1932), the debate has generally focused on selection factors versus environmental factors to explain higher rates of severe mental illness among migrants. In the present study we are seeking to explain lower rates among the migrants across a broad spectrum of common psychiatric disorders. The two principal explanations for our findings are that: the more healthy migrate to the cities; and urban living is more conducive to good mental health than the rural environment in Pakistan.
Individuals and families who migrate to the cities may be psychologically more resourceful and resilient than those they leave behind. However, in the Pakistani context, the decision to migrate is made principally by the senior male head of the family in the village or sometimes by the head of the migrating household (usually one of his sons). The women of the family often have little influence on the decision to migrate, yet they also enjoy much better mental health than women back in the village.

The new urban environment may contribute to an improvement in mental health. Life in the city is much less constrained, both socially and economically, than in the village. There is more choice with regard to work, friends, schools and social activities. Earnings are much higher, offering greater material prosperity, wider consumer choice and the opportunity to fulfil personal ambitions. Physical health may also be better in cities than in villages, partly as a result of access to better medical facilities.

Two of the authors of the present study (I.A. and S.A.) have direct experience of this migration process within their own families in Pakistan. Their fathers migrated from their villages into towns in order to obtain better education for their immediate families, which opened up for them the possibility of a university education and a professional career in medicine. Not all the family group has migrated, however, and they still have uncles and cousins who remain in the ancestral village. Some of the observed rural-urban discrepancy in common psychiatric disorders may perhaps reflect frustration and discontent among those of the joint/extended family who remain behind and are denied the opportunities offered in the city.

However, these explanations remain speculative. We need to conduct more detailed family studies of internal migration and its effects. There is often a considerable degree of mobility between branches of the same joint/extended family resident in the city and in the village. This will facilitate case-control studies of migrants with varying lengths of exposure to the urban environment, and also cohort studies of migrant branches of families using members of the same families left behind in the ancestral village for comparison. Greater understanding of the impact on mental health of migration into the cities of 
Pakistan may help to shed light on the factors behind the poor mental health status of people who live in rural areas.

\section{ACKNOWLEDGEMENTS}

The authors thank the residents of Sultanpura who offered assistance and hospitality during this study, especially Raja Matloob Abbassi and Mr Sala-uddin, and also Rabia, Vanus, ljaz Kaleem and Ishaq, who administered the screening questionnaires.

\section{REFERENCES}

Almeida-Filho, N. (1987) Social epidemiology of mental disorders: a review of Latin American studies. Acta Psychiatrica Scandinavica, 75, I-10.

American Psychiatric Association (1980) Diagnostic and Statistical Manual of Mental Disorders (3rd edn) (DSM-III). Washington, DC: APA.

Brown, G.W. \& Harris, T. O. (1978) Social Origins of Depression. London: Tavistock.

— \& Prudo, R. (198I) Psychiatric disorder in a rura and an urban population. I. Aetiology of depression. Psychological Medicine, II, 58I-599.

Canino, G. J., Bird, H. R., Shrout, P. E., et al (1987) The prevalence of specific psychiatric disorders in Puerto Rico. Archives of General Psychiatry, 44, 727-735.

Cattell, R. B. (1966) The meaning and strategic use of factor analysis. In Handbook of Multivariate Experimental Psychology (ed. R. B. Cattell). Chicago: Rand McNally.

Cheng, T. A. (1989) Urbanisation and minor psychiatric morbidity. Social Psychiatry and Psychiatric Epidemiology, 24, 309-316.

Desjarlais, R., Eisenberg, L., Good, B., et al (1995) World Mental Health: Problems and Priorities in LowIncome Countries. New York: Oxford University Press.

Dube, K. C. (1970) A study of prevalence and biosocial variables in mental illness in a rural and an urban community in Uttar Pradesh, India. Acta Psychiatrica Scandinavica, 46, 327-359.

Guarnaccia, P. J., Good, B. J. \& Kleinman, A. (1990) A critical review of epidemiological studies of Puerto Rican mental health. American Journal of Psychiatry, 147 1449-1456.

Harpham, T. (1992) Urbanisation and mental disorder In Principles of Social Psychiatry (eds D. Bhugra \& J. Leff) pp. 346-354. New York: Blackwell.

\section{Hwu, H.-G., Yeh, E.-K. \& Chang, L.-Y. (1989)}

Prevalence of psychiatric disorders in Taiwan defined by the Chinese Diagnostic Interview Schedule. Acta Psychiatrica Scandinavica, 79, 136-147.

Lee, C. K., Kwak, Y. S., Yamonto, J., et al (1990) Psychiatric epidemiology in Korea. Part II: Urban and rural differences. Journal of Nervous and Mental Disease, I78, $247-252$

Mari, J. (1997) Psychiatric morbidity in three primary medical care clinics in the city of Sao Paulo. Socia Psychiatry, 22, 129-138.

Mumford, D. B. (1992) The Development and Validation of the Bradford Somatic Inventory: an Investigation of

\section{CLINICAL IMPLICATIONS}

- People who live in some urban areas of Pakistan may carry a lower risk of common mental disorders than those living in nearby rural areas. It may be useful for psychiatrists in Britain to enquire about the rural/urban origin of first-generation migrants from Pakistan.

- Enquiring about psychological symptoms in non-Western patients who present initially with somatic symptoms is worthwhile, in their own language and respecting local cultural idioms of distress.

- An inventory consisting only of somatic symptoms is effective in screening for common mental disorders, and may have advantages in cultures where most people with psychiatric disorders express their distress in somatic complaints.

\section{LIMITATIONS}

- At the interview stage, time constraints required us to select random samples of men and women in the sub-threshold Bradford Somatic Inventory scoring category.

- Although the study identified certain subgroups at higher risk of common mental disorders (e.g. women living in joint households), we did not obtain qualitative data that might shed light on the reasons for this increased risk.

- It was not possible in this study to establish whether the lower rates of common mental disorders in an urban area compared with nearby rural areas were due to selective migration or to environmental factors.

DAVID BARDWELL MUMFORD, MRCPsych, Division of Psychiatry, University of Bristol, Bristol, UK; FAREED ASLAM MINHAS, MRCPsych, IMTIAZ AKHTAR, MBBS, SAEED AKHTER, MBBS, MALIK HUSSAIN MUBBASHAR, FRCPsych, Institute of Psychiatry, Rawalpindi Medical College, Pakistan

Correspondence: David B. Mumford, Division of Psychiatry, University of Bristol, 4I St Michael's Hill, Bristol BS2 8DZ, UK. Tel: 01179287773 ; fax: 01179259709

(First received 10 December 1999, final revision 7 June 2000, accepted 9 June 2000)

Functional Somatic Symptoms in Britain and Pakistan. MD Thesis, University of Bristol.

_ , Bavington, J.T., Bhatnagar, K. S., et al (1991) The Bradford Somatic Inventory. A multi-ethnic inventory of somatic symptoms reported by anxious and depressed patients in Britain and the Indo-Pakistan subcontinent. British Journal of Psychiatry, 158, 379-386.

_, Nazir, M., Jilani, F. M., et al (1996) Stress and psychiatric disorder in the Hindu Kush: a community survey of mountain villages in Chitral, Pakistan. British Journal of Psychiatry, 168, 299-307.

_ , Saeed, K., Ahmad, I., et al (1997) Stress and psychiatric disorder in rural Punjab: a community survey British Journal of Psychiatry, 170, 473-478.

Odegaard, O. (1932) Emigration and insanity. Acta Psychiatrica et Neurologica Scandinavica, 7 (suppl. 4), 206.
Regier, D. A. \& Kaelber, C. T. (1995) The Epidemiologic Catchment Area (ECA) Program: studying the prevalence and incidence of psychopathology. In Textbook in Psychiatric Epidemiology (eds M. T. Tsuang, M. Tohen \& G. E. P. Zahner), pp. 135-155. New York: Wiley-Liss.

Sethi, B. B., Gupta, S. C., Mahendru, R. K., et a (1974) Mental health and urban life: a study of 850 families. British Journal of Psychiatry, 124, 243-246.

Wing, J. K., Cooper, J. E. \& Sartorius, N. (1974) The Measurement and Classification of Psychiatric Symptoms. Cambridge: Cambridge University Press.

World Health Organization (1993) The ICD- 10 Classification of Mental and Behavioural Disorders: Diagnostic Criteria for Research. Geneva: WHO. 